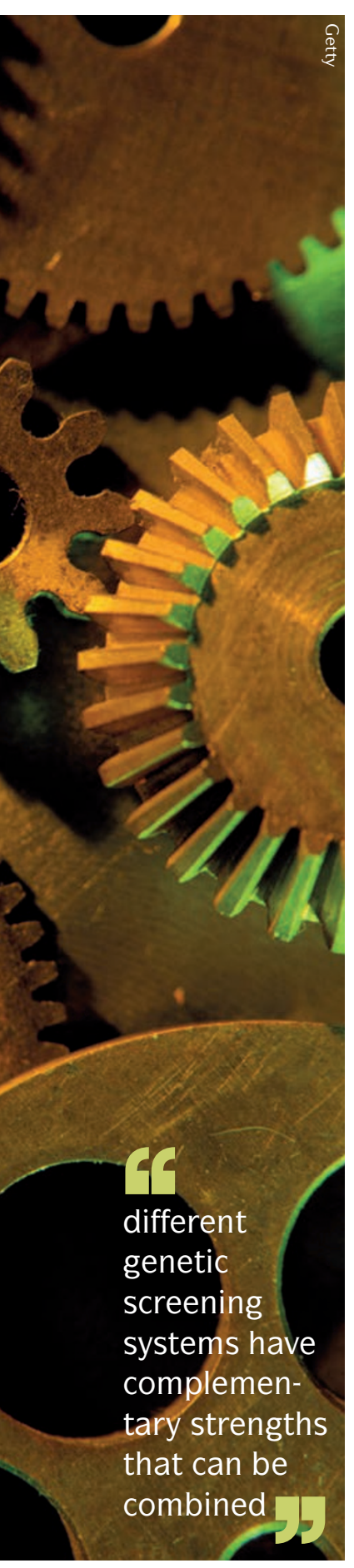

$\Rightarrow$ GENETIC SCREENS

\title{
Combination screens for combination therapies
}

Resources for genome-scale loss-offunction screens in mammalian cells have progressed rapidly, particularly with RNA interference (RNAi)based libraries and, more recently, with CRISPR-Cas9-based libraries. A new parallel screening study leverages the complementary strengths of each system to dissect antiviral drug mechanisms and to identify potential combination therapy strategies.

Deans et al. sought to dissect the mechanism of action of the antiviral small molecule GSK983. This compound is thought to act by inhibiting a host factor that is involved in both viral replication and host physiology, as the antiviral effects are accompanied by host cell toxicity. Thus, identifying the actual molecular target and understanding the mechanism of action of the compound could point to ways to minimize host cytotoxicity while retaining antiviral activity.

To identify which host factors are involved in the drug response, the investigators used two genome-scale screening strategies in human K562 cells. The first was an RNAi library targeting all human protein-coding genes using $\sim 25$ short hairpin RNAs (shRNAs) per gene. These were delivered lentivirally into cells and relied on the endogenous cellular RNAi machinery for knockdown. The second was a CRISPR-based library targeting all protein-coding genes using $\sim 4$ single-guide RNAs (sgRNAs) per gene. This sgRNA library was lentivirally delivered into a K562 cell line stably expressing bacterial Cas9 nuclease; target genes are genetically inactivated following Cas9-mediated double-strand breaks and mutagenic non-homologous end-joining repair.

In both screens, pools of library-transduced cells were either treated with GSK983 or left untreated. The shRNA or sgRNA constructs that sensitize to or protect from GSK983 treatment will alter cell survival and thus should become relatively depleted or enriched, respectively, in the treated cell population. Such effects were read out using high-throughput sequencing to quantify the genomic copy numbers of each library construct in the cell populations.

The team focused on shRNAs and sgRNAs that caused the most substantial alterations to GSK983 sensitivity. Although the gene hits overlapped between the shRNA and sgRNA screens, hence pointing to some common pathways, there were also notable differences. The shRNA screen identified that knockdown of the pyrimidine biosynthesis gene dihydroorotate dehydrogenase $(D H O D H)$ sensitized cells to GSK983. Such behaviour is expected of the drug target, as lower expression of the target protein will require less drug-mediated inhibition to reduce the overall enzyme activity to below a cytotoxic threshold. Follow-up experiments, including biochemical assays of recombinant DHODH activity in vitro, provided additional support for DHODH as the drug target. $\mathrm{DHODH}$ is an essential gene and was not identified in the sgRNA screen, probably because complete DHODH knockout is incompatible with cell viability regardless of drug treatment.

By contrast, only the sgRNA screen identified that loss of various components of mTOR signalling, including NPR2-like GATOR1 complex subunit (NPRL2), sensitized cells to GSK983. These genes are likely to require complete loss of function, rather than incomplete knockdown, for these phenotypic effects to be seen.
Overall, the screens found multiple genes involved in pyrimidine biosynthesis and salvage pathways. Hence, the authors reasoned that drug-mediated disruption to pyrimidine metabolism for viral and host nucleic acids was the probable cause of both the antiviral and cytotoxic effects. To separate these effects, the team tested a combination of GSK983 and deoxycytidine, with the aim that deoxycytidine will act as a salvage metabolite to support host DNA replication during GSK983 treatment, without salvaging replication of RNA viruses. Indeed, deoxycytidine reduced GSK983 cytotoxicity, while retaining its antiviral activity towards Dengue virus and Venezuelan equine encephalitis virus in infected human cultured cells.

This study highlights how different genetic screening systems have complementary strengths that can be combined. CRISPR-based knockout screens can achieve the complete loss of function that is required for dissecting the roles of some genes. RNAi-based knockdown screens can interrogate the functions of essential genes, and shRNAs will remain as valuable tools for pharmacological applications, as incomplete knockdown may provide a closer mimic of pharmacological inhibition than does complete and irreversible genetic knockout. It will be interesting to see the impact of parallel screening strategies in diverse biological applications.

Darren J. Burgess

ORIGINAL ARTICLE Deans, R. M. et al. Parallel shRNA and CRISPR-Cas9 screens enable antiviral drug target identification. Nat. Chem. Biol. http:// dx.doi.org/10.1038/nchembio.2050 (2016) FURTHER READING Shalem, O., Sanjana, N. E. \& Zhang, F. High-throughput functional genomics using CRISPR-Cas9. Nat. Rev. Genet. 16, 299-311 (2015) 